\title{
Energy-Information Tradeoffs in Motion and Sensing for Target Localization
}

\author{
Wencen Wu, Fumin Zhang, and Yorai Wardi
}

\begin{abstract}
Recent study in behavioral ecology reveal that certain species trade-off energy and information during various routine activities. This paper explores a similar tradeoff between sensing and motion of mobile agents tasked with target localization. Specifically, the goal is to balance the quality of information collected by sensing agents with the kinetic energy expended by their motion relative to the target. Following common practice, the determinant of the Fisher Information Matrix is chosen to represent the information-quality, while the kinetic energy is quantified by the usual Newton's laws. We argue that the quality of information has a form reminiscent of potential energy, and with such interpretation, we mimic the basic laws of mechanics by assuming the conservation of the total energy, which is the sum of the potential energy and kinetic energy. In this framework, we formulate an optimal path problem that aims at increasing the information quality while reducing the kinetic energy along the path of the agents. To solve this problem, we apply the calculus of variations, only to discover that the optimal solutions (paths) satisfy the EulerLagrange equations that are well known in classical mechanics. This realization suggests that the resulting trajectories may exhibit some well-known phenomena in classical mechanics. Simulation results support this hypothesis.
\end{abstract}

\section{INTRODUCTION}

In robotics research, target localization is an important research topic with a wide range of civilian and military applications such as reconnaissance, surveillance, and environmental mapping [1], [2]. These applications require the co-design of sensing and motion of agents that operate in an environment with large motion disturbances and information uncertainties [3]-[5]. Mobile sensor networks are routinely used for such application problems since measurements from multiple sensors can be combined and filtered to produce efficient and reliable state estimation [6]-[10].

Energy-information tradeoff is a general principle for biological mobile sensing. The authors in [11] observe that when searching for prey, electric fish swim in a less efficient manner in order to achieve a higher encounter rate for prey. The electric fish is also found to move longer distance in darkness to track a moving target when the visual information is absent and the electrosensory information is degraded [12]. Tradeoffs between information and energy are also observed in bees. For example, nocturnal bees fly at lower speeds compared to diurnal bees. However, their

The research work is supported by ONR grants N00014-08-11007, N00014-09-1-1074, and N00014-10-10712 (YIP), and NSF grants ECCS-0841195 (CAREER), CNS-0931576, and ECCS-1056253. Wencen Wu, Fumin Zhang, and Yorai Wardi are with the School of Electrical and Computer Engineering, Georgia Institute of Technology, Atlanta, GA, 30332, USA wwencen3, fuminegatech.edu, yorai.wardidece.gatech.edu apposition eyes are 30 times more optically-sensitive than the eyes of their closest diurnal relatives [13], [14]. Research on the tradeoffs between speed in decision making and accuracy in obtaining information in animals are reviewed in [15]. Decision making speed can be related to the energy consumption in certain cases.

Inspired by such biological insights, we investigate the tradeoffs between energy and information of mobile agents for target localization. Energy consumption in mobile sensor networks is an important issue that determines the operation time in real-life applications. The major contributors to energy consumption include motion, communication, and sensor information processing. Sensor scheduling and communication scheduling have been intensively studied in wireless sensor networks. For mobile sensor networks, motion usually is the largest user of energy, and hence we focus on balancing the kinetic energy consumption of the agents and the quality of information collected by them.

Most existing works on trajectory optimization for target localization are based on maximizing the information obtained by sensing agents. For example, the Fisher information matrix (FIM), which describes the amount of information that the measurements carry about the states of the target, is widely used as a criteria for performance measure and trajectory optimization [1], [16]-[21]. In these works, the speed of the agents is usually assumed to be constant, which implies constant kinetic energy of the agents. Our approach is new since instead of just maximizing the information, we aim at balancing the tradeoffs between information and energy.

Our basic idea is underscored by a realization that the quality of information, as characterized by the Fisher information matrix, is akin to a measure of potential energy. The reason is that the determinant of the FIM only contains spatial (geometric) information about the agents, not unlike measures of potential energy in models of mechanical systems. The objective of this paper is to initiate an exploration of the balance between high information quality and low kinetic energy in this setting; we do so by defining an optimalpath problem whose objective is to minimize the difference between the agents' kinetic energy and the potential energy, which is represented by the inverse of the informationquality. By viewing the agents as free Newtonian particles subjected to only the potential forces introduced by potential functions, the resulting trajectories are known to satisfy the Euler-Lagrange equations, or the Hamilton equations [22]. Furthermore, borrowing a fundamental idea from classical mechanics, we assume that the total energy of the system is conserved along the trajectories of the mobile agents. As 
a first step, we consider two agents tracking one stationary target, and derive the Hamilton equations for them. We then present simulation results for various initial conditions.

The rest of the paper is organized as follows. Section II presents background information on bearings-only target localization using mobile sensing agents. Section III discusses the energy-information tradeoffs in target localization and formulates an optimal control problem. Section IV introduces the solutions for the trajectories of the agents derived from Hamilton equations. Section V demonstrates simulation results, and Section VI concludes the paper.

\section{BEARINGS-ONLY TARGET LOCALIZATION}

Bearings-only target motion analysis (TMA) is a classical problem, in which an observer measures noisy bearings from a target and estimates the position and velocity of the target [23], [24]. In this section, we formulate the tracking problem when $N$ agents are deployed to localize a target using bearings-only measurements and the Fisher information matrix (FIM) associated with this problem.

\section{A. Tracking Model}

Consider a team of $N$ sensing agents that are moving in a 2D field to localize a target with bearings-only measurements. Denote the position of the target as $\mathbf{r}_{T}=\left(r_{T, x}, r_{T, y}\right)$, the position of the $i$ th agent as $\mathbf{r}_{i}=\left(r_{i, x}, r_{i, y}\right)$, and the corresponding bearing measurement as $\theta_{i}, i=1, \cdots, N$. Assume that both the target and the agents are point masses, which satisfy $\dot{\mathbf{r}}_{T}=\mathbf{v}_{T}$, and $\dot{\mathbf{r}}_{i}=\mathbf{v}_{i}, i=1, \cdots, N$, where $\mathbf{v}_{T}$ and $\mathbf{v}_{i}$ are the velocities of the target and the $i$ th agent, respectively.

Fig. 1 illustrates the geometry of two agents tracking a target, in which $d_{i}$ is the distance and $\theta_{i}$ is the bearing angle from the $i$ th agent to the target. The bearing angle $\theta_{i}$ is related to the relative position between the target and the agent by

$$
\theta_{i}=\arctan \frac{r_{T, y}-r_{i, y}}{r_{T, x}-r_{i, x}} .
$$

Define $h(\mathbf{s})$ as $h(\mathbf{s})=\left[\theta_{1}, \cdots, \theta_{N}\right]^{T}$, in which $\mathbf{s}=\mathbf{r}_{T}^{T}=$ $\left(r_{T, x}, r_{T, y}\right)^{T}$ is the state vector of the target. Let $\mathbf{y}$ be the measurement vector consisting of all the measurements collected from the $N$ collaborating agents. Then, the measurement equation for the $N$ sensing agents is

$$
\mathbf{y}(t)=h(\mathbf{s}(t))+\mathbf{w}(t),
$$

where $\mathbf{w} \sim \mathscr{N}(0, R)$ is assumed to be zero-mean Gaussian noise with covariance matrix $R$. Therefore, the measurement vector $\mathbf{y}$ is a normally distributed random vector with mean $h(\mathbf{s})$ and covariance matrix $R$, i.e., $\mathbf{y} \sim \mathscr{N}(h(\mathbf{s}), R)$.

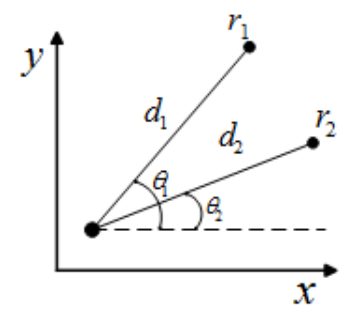

Fig. 1. Geometry of two agents tracking a target.

\section{B. Fisher Information}

The Fisher information matrix (FIM) describes the amount of information that the measurement $\mathbf{y}$ carries about the state s. It is calculated that, given the measurement equation (2), the FIM is [3]

$$
\mathrm{I}(\mathbf{s})=\nabla_{\mathbf{s}} h(\mathbf{s})^{T} R^{-1} \nabla_{\mathbf{s}} h(\mathbf{s}) .
$$

Define the distance from the target to the $i$ th sensing agent as $d_{i}=\left\|\mathbf{r}_{T}-\mathbf{r}_{i}\right\|$. For a stationary target, $d_{i}=\left\|\mathbf{r}_{i}\right\|$.

From the measurement equation (2), we derive that

$$
\mathrm{I}(\mathbf{s})=\frac{1}{\sigma_{w}^{2}}\left(\begin{array}{cc}
\sum_{i=1}^{N} \frac{1}{d_{i}^{2}} \sin ^{2} \theta_{i} & -\sum_{i=1}^{N} \frac{1}{2 d_{i}^{2}} \sin 2 \theta_{i} \\
-\sum_{i=1}^{N} \frac{1}{2 d_{i}^{2}} \sin 2 \theta_{i} & \sum_{i=1}^{N} \frac{1}{d_{i}^{2}} \cos ^{2} \theta_{i}
\end{array}\right),
$$

where $\sigma_{w}^{2}$ is the strength of the noise covariance matrix $R$. That is, $R=\sigma_{w}^{2} I$, in which $I$ is an identity matrix.

The Cramer-Rao lower bound (CRLB) indicates that the variance of any unbiased estimator is bounded below by the inverse of the determinant of Fisher information matrix. As stated in [3], [19]-[21], a configuration of the agents over the space of all angle positions $\theta_{i}, \forall i \in\{1, \cdots, N\}$ is optimal if the configuration maximizes the determinant of Fisher information matrix, or minimizes the Cramer-Rao lower bound. The determinant of the FIM can be calculated as

$$
\operatorname{det}(\mathrm{I})=\frac{1}{\sigma_{\mathrm{w}}^{2}} \sum_{\{\mathrm{i}, \mathrm{j}\}} \frac{\sin ^{2}\left(\theta_{\mathrm{i}}-\theta_{\mathrm{j}}\right)}{\mathrm{d}_{\mathrm{i}}^{2} \mathrm{~d}_{\mathrm{j}}^{2}}, \mathrm{i}>\mathrm{j} .
$$

For $N=2$, the determinant is simplified to

$$
\operatorname{det}(\mathrm{I})=\frac{\sin ^{2}\left(\theta_{2}-\theta_{1}\right)}{\sigma_{\mathrm{w}}^{2} \mathrm{~d}_{1}^{2} \mathrm{~d}_{2}^{2}},
$$

indicating that to obtain maximum information in tracking a target, the two agents should triangulate, i.e., $\theta_{2}-\theta_{1} \rightarrow \frac{\pi}{2}$ and move towards the target, i.e., $d_{1}, d_{2} \rightarrow 0$.

\section{ENERGY-INFORMATION TRADEOFFS}

Target localization requires the co-design of motion and sensing for mobile sensing agents. We aim at balancing the information and energy of the agents. In this section, we introduce the kinetic and potential energy of the system that two agents are tracking a stationary target and demonstrate the tradeoffs between information and energy of the tracking system.

In mechanical systems, the total energy consists of both kinetic energy and potential energy. The total kinetic energy of the agents is

$$
E=\frac{1}{2} \sum_{i=1}^{N} m_{i} v_{i}^{2}
$$

where $v_{i}$ is the speed and $m_{i}$ is the mass of the $i$ th agent. Since we consider the angle $\theta_{i}$ and distance $d_{i}$ in the FIM, we use polar coordinates to present the kinetic energy as well. Let us consider the case of two agents, as shown in Fig. 1. 
Suppose the agents locates at $(0,0)$. Then, the positions of the agents becomes

$$
\begin{aligned}
& r_{1, x}=d_{1} \cos \theta_{1}, \\
& r_{1, y}=d_{1} \sin \theta_{1}, \\
& r_{2, x}=d_{2} \cos \theta_{2}, \\
& r_{2, y}=d_{2} \cos \theta_{2} .
\end{aligned}
$$

from which we obtain the speed of the agents by taking derivatives with respect to time,

$$
\begin{aligned}
& v_{1}^{2}=\dot{r}_{1, x}^{2}+\dot{r}_{1, y}^{2}=\dot{d}_{1}^{2}+d_{1}^{2} \dot{\theta}_{1}^{2}, \\
& v_{2}^{2}=\dot{r}_{2, x}^{2}+\dot{r}_{2, y}^{2}=\dot{d}_{2}^{2}+d_{2}^{2} \dot{\theta}_{2}^{2} .
\end{aligned}
$$

Notice that the difference in the bearing angles $\theta_{1}-\theta_{2}$ and the distances $d_{1}$ and $d_{2}$ between the agents and the target actually describe a formation maintained by the target and the two agents. Therefore, we define shape variables $\left(d_{1}, d_{2}, \gamma\right)$, in which $\gamma=\frac{1}{2}\left(\theta_{1}-\theta_{2}\right)$, to represent the formation. In addition, it will be convenient to define the variable $\phi:=$ $\frac{1}{2}\left(\theta_{1}+\theta_{2}\right)$ to describe the orientation of the formation. Then, we have $\dot{\gamma}=\frac{1}{2}\left(\dot{\theta}_{1}-\dot{\theta}_{2}\right), \dot{\phi}=\frac{1}{2}\left(\dot{\theta}_{1}+\dot{\theta}_{2}\right), \theta_{1}=\gamma+\phi$, and $\theta_{2}=\phi-\gamma$. Substitute $\theta_{1}$ and $\theta_{2}$ into equations (9), we obtain

$$
\begin{aligned}
& v_{1}^{2}=\dot{d}_{1}^{2}+d_{1}^{2}\left(\dot{\gamma}^{2}+2 \dot{\gamma} \dot{\phi}+\dot{\phi}^{2}\right), \\
& v_{2}^{2}=\dot{d}_{2}^{2}+d_{2}^{2}\left(\dot{\phi}^{2}-2 \dot{\gamma} \dot{\phi}+\dot{\gamma}^{2}\right) .
\end{aligned}
$$

Assume that both agents have unit mass, i.e., $m_{1}=m_{2}=1$. Then, the total kinetic energy of the two agents is

$$
\begin{aligned}
& E=v_{1}^{2}+v_{2}^{2} \\
& =\frac{1}{2}\left(\dot{d}_{1}^{2}+\dot{d}_{2}^{2}+\left(d_{1}^{2}+d_{2}^{2}\right) \dot{\phi}^{2}+2\left(d_{1}^{2}-d_{2}^{2}\right) \dot{\gamma} \dot{\phi}+\left(d_{1}^{2}+d_{2}^{2}\right) \dot{\gamma}^{2}\right) .
\end{aligned}
$$

For measuring the quality of information collected by the agents, we choose the determinant of the FIM, as shown in Equation (6), and aim at maximize the determinant along the trajectories of agents. Since the determinant of FIM only contains spatial (geometric) information of the agents, i.e., $\theta_{i}$ and $d_{i}$, it is akin to a measure of potential energy. Denote

$$
V_{I}=\frac{K_{1} d_{1}^{2} d_{2}^{2}}{\sin ^{2}(2 \gamma)},
$$

which is the inverse of the determinant of FIM. And design

$$
V_{A}=\frac{K_{2}}{2}\left(\frac{1}{d_{1}^{2}}+\frac{1}{d_{2}^{2}}\right)
$$

where $K_{1}$ and $K_{2}$ are constants. Then, we define the potential energy of the system as

$$
V=V_{I}+V_{A} .
$$

Equation (12) indicates that, when the agents are close to the target, i.e., $d_{i} \rightarrow 0$, and when $\gamma \rightarrow \frac{\pi}{4}, V_{I}$ is minimized, which maximize the determinant of FIM. However, collision between the agents and the target needs to be avoided. Therefore, the repelling force $V_{A}$ is introduced since as $d_{i} \rightarrow$ $0, V_{A} \rightarrow \infty$. By defining the kinetic energy $E$ and potential energy $V$, the total energy of the system is $E+V$.
It has been known that when two agents are localizing one target, the two agents tend to triangulate and move towards the target as quickly as possible [1], [18] since $\gamma=\frac{\pi}{4}$ and $d_{i}=0, i=1,2$ would maximize the determinant of FIM. However, if the agents travel with maximum speed all the time along their trajectories, they consume maximum kinetic energy during a certain time period. This result agrees with the observation of the behaviors of electric fish as described in [11]. When an electric fish consumes more energy during a certain time period, it obtains more information. However, in real-world applications of target tracking problem, we aim to reduce the energy consumed by the agents, which is represented by the kinetic energy $E$. Meanwhile, we want to gather more information during a certain time period, which is represented by the determinant of FIM denoted by $\frac{1}{V_{I}}$. This argument indicates a tradeoff between the kinetic energy and the information collected by the agents.

\section{Optimal Trajectories of the Mobile Agents}

In this section, we formulate an optimal path problem to address the tradeoffs between kinetic energy and potential energy and provide solutions of the optimal path problem using Hamilton equations. The following assumption holds for the rest of the paper.

Assumption 4.1: The mobile agents are assumed to be free Newtonian particles subjected to only the potential forces introduced by the potential function $V(\mathbf{q}(t))$.

\section{A. Cost Function}

Let $\mathbf{q}=\left(q_{1}, \cdots, q_{n}\right)^{T}$ represent the generalized coordinates for the positions of the agents and $\dot{\mathbf{q}}$ represent the generalized velocities. Suppose $(\mathbf{q}, \dot{\mathbf{q}})$ satisfies $\ddot{\mathbf{q}}=f(\mathbf{q}, \dot{\mathbf{q}}, \mathbf{u})$, where $\mathbf{u}$ represents the total control effort. In the target localization problem, we define $q_{1}=d_{1}, q_{2}=d_{2}, q_{3}=\gamma$, and $q_{4}=\phi$. Then, we define an integrated cost function to be

$$
J(\mathbf{q}, \dot{\mathbf{q}})=\int_{0}^{T}(E(\mathbf{q}(t), \dot{\mathbf{q}}(t))-V(\mathbf{q}(t))) d t,
$$

where $T$ denotes a given terminal time. We aim to find the extrema of the cost function subjecting to some control forces. Solutions of the optimal control problem provide optimal trajectories for mobile sensing agents.

Under Assumption 4.1, the dynamic constraint $\ddot{\mathbf{q}}=$ $f(\mathbf{q}, \dot{\mathbf{q}}, \mathbf{u})$ is not present. In this case, the optimal solution is known to satisfy the Euler-Lagrange equation, or Hamilton equation [22]. The total energy of the system $H=E+V$, in which $H$ is recognized as the Hamiltonian of the system is always conserved along the trajectories of the mobile agents.

The cost function (15) indicates that, to obtain the minimum of the kinetic energy, $V$ needs to be maximized, suggesting that the information represented by $\frac{1}{V}$, is also minimized. This fact agrees with the biological inspirations. However, due to the conservation of the energy, a balance between the kinetic energy and potential energy should be found to obtain the optimal trajectories of the agents. 


\section{B. Solutions Derived from the Hamilton Equations}

In Hamiltonian mechanics, $L(\mathbf{q}(t), \dot{\mathbf{q}}(t))=E(\mathbf{q}(t), \dot{\mathbf{q}}(t))-$ $V(\mathbf{q}(t))$ is the Lagrangian of the system. The first order necessary condition for optimality is the solution of the Euler-Lagrange equation

$$
\frac{d}{d t}\left(\frac{\partial L}{\partial \dot{\mathbf{q}}}\right)-\frac{\partial L}{\partial \mathbf{q}}=0
$$

The Hamiltonian equations can be derived from the Lagrangian equations in that it translates $n$ second-order differential equations describing the motion of a particle into $2 n$ first-order equations, where $n$ is the number of degrees of freedom of the system [22]. Define the generalized momenta as $\mathbf{p}=\frac{\partial L}{\partial \dot{\mathbf{q}}}$. The Hamiltonian and the Lagrangian are related by the Legendre transformation $\dot{\mathbf{q}}=h(\mathbf{q}, \mathbf{p}, t)$ as follows

$$
H(\mathbf{q}, \mathbf{p}, t)=\mathbf{p}^{T} h(\mathbf{q}, \mathbf{p}, t)-L(\mathbf{q}, h(\mathbf{q}, \mathbf{p}, t), t),
$$

which produces the Hamilton equations as

$$
\dot{\mathbf{p}}=-\frac{\partial H}{\partial \mathbf{q}}, \quad \dot{\mathbf{q}}=\frac{\partial H}{\partial \mathbf{p}} .
$$

The trajectories of the agents can be obtained by solving the above two partial differential equations.

In the special case when two agents track a stationary target, the potential energy is

$$
V(\mathbf{q})=V_{I}(\mathbf{q})+V_{A}(\mathbf{q})=\frac{K_{1} q_{1}^{2} q_{2}^{2}}{\sin ^{2}\left(2 q_{3}\right)}+\frac{K_{2}}{2}\left(\frac{1}{q_{1}^{2}}+\frac{1}{q_{2}^{2}}\right),
$$

and the kinetic energy is

$$
\begin{aligned}
& E(\mathbf{q}, \dot{\mathbf{q}}) \\
& =\frac{1}{2}\left(\dot{q}_{1}^{2}+\dot{q}_{2}^{2}+\left(q_{1}^{2}+q_{2}^{2}\right) \dot{q}_{4}^{2}+2\left(q_{1}^{2}-q_{2}^{2}\right) \dot{q}_{3} \dot{q}_{4}+\left(q_{1}^{2}+q_{2}^{2}\right) \dot{q}_{3}^{2}\right) .
\end{aligned}
$$

Therefore, the cost function can be written as

$$
\begin{aligned}
& J(\mathbf{q}, \dot{\mathbf{q}})=\int_{0}^{t} L(\mathbf{q}, \dot{\mathbf{q}}, t) d t \\
& =\int_{0}^{t} \frac{1}{2}\left(\dot{q}_{1}^{2}+\dot{q}_{2}^{2}+\left(q_{1}^{2}+q_{2}^{2}\right) \dot{q}_{3}^{2}+2\left(q_{1}^{2}-q_{2}^{2}\right) \dot{q}_{3} \dot{q}_{4}+\left(q_{1}^{2}+q_{2}^{2}\right) \dot{q}_{4}^{2}\right) \\
& -\frac{K_{1} q_{1}^{2} q_{2}^{2}}{\sin ^{2}\left(2 q_{3}\right)}-\frac{K_{2}}{2}\left(\frac{1}{q_{1}^{2}}+\frac{1}{q_{2}^{2}}\right) d t .
\end{aligned}
$$

To solve for the trajectories of the agents, we first calculate the general momenta $\mathbf{p}=\frac{\partial L}{\partial \dot{\mathbf{q}}}$ as

$$
\begin{aligned}
& p_{1}=\dot{q}_{1}, \\
& p_{2}=\dot{q}_{2}, \\
& p_{3}=\left(q_{1}^{2}+q_{2}^{2}\right) \dot{q}_{3}+\left(q_{1}^{2}-q_{2}^{2}\right) \dot{q}_{4}, \\
& p_{4}=\left(q_{1}^{2}+q_{2}^{2}\right) \dot{q}_{4}+\left(q_{1}^{2}-q_{2}^{2}\right) \dot{q}_{3},
\end{aligned}
$$

which yields the Legendre transform $\dot{\mathbf{q}}=h(\mathbf{q}, \mathbf{p}, t)$,

$$
\begin{aligned}
& \dot{q}_{1}=p_{1}, \\
& \dot{q}_{2}=p_{2}, \\
& \dot{q}_{3}=\frac{\left(q_{1}^{2}+q_{2}^{2}\right) p_{3}-\left(q_{1}^{2}-q_{2}^{2}\right) p_{4}}{4 q_{1}^{2} q_{2}^{2}}, \\
& \dot{q}_{4}=\frac{\left(q_{1}^{2}+q_{2}^{2}\right) p_{4}-\left(q_{1}^{2}-q_{2}^{2}\right) p_{3}}{4 q_{1}^{2} q_{2}^{2}} .
\end{aligned}
$$

Then, according to Equation (17), the Hamiltonian is

$$
\begin{aligned}
& H(\mathbf{q}, \mathbf{p}, t)=\frac{1}{2}\left(p_{1}^{2}+p_{2}^{2}+\frac{\left(p_{3}+p_{4}\right)^{2}}{4 q_{1}^{2}}+\frac{\left(p_{3}-p_{4}\right)^{2}}{4 q_{2}^{2}}\right) \\
& +\frac{K_{1} q_{1}^{2} q_{2}^{2}}{\sin ^{2}\left(2 q_{3}\right)}+\frac{K_{2}}{2}\left(\frac{1}{q_{1}^{2}}+\frac{1}{q_{2}^{2}}\right) .
\end{aligned}
$$

Therefore, $\dot{\mathbf{p}}=-\frac{\partial H}{\partial \mathbf{q}}$ can be calculated as

$$
\begin{aligned}
& \dot{p}_{1}=\frac{1}{4 q_{1}^{3}}\left(p_{3}+p_{4}\right)^{2}+\frac{K_{2}}{q_{1}^{3}}-\frac{2 K_{1} q_{1} q_{2}^{2}}{\sin ^{2}\left(2 q_{3}\right)}, \\
& \dot{p}_{2}=\frac{1}{4 q_{2}^{3}}\left(p_{3}-p_{4}\right)^{2}+\frac{K_{2}}{q_{2}^{3}}-\frac{2 K_{1} q_{1}^{2} q_{2}}{\sin ^{2}\left(2 q_{3}\right)}, \\
& \dot{p}_{3}=\frac{4 K_{1} q_{1}^{2} q_{2}^{2} \cos \left(2 q_{3}\right)}{\sin ^{3}\left(2 q_{3}\right)}, \\
& \dot{p}_{4}=0 .
\end{aligned}
$$

The optimal trajectories are the solution of Equations (23) and (25) with given initial conditions $\mathbf{q}\left(t_{0}\right)$ and $\mathbf{p}\left(t_{0}\right)$.

We observe from Equation (25) that $p_{4}=C$, in which $C$ is a constant. Then, the number of differential equations in (23) and (25) can be reduced from 8 to 6 , that is,

$$
\begin{aligned}
& \dot{q}_{1}=p_{1}, \\
& \dot{q}_{2}=p_{2}, \\
& \dot{q}_{3}=\frac{\left(q_{1}^{2}+q_{2}^{2}\right) p_{3}-\left(q_{1}^{2}-q_{2}^{2}\right) C}{4 q_{1}^{2} q_{2}^{2}}, \\
& \dot{p}_{1}=\frac{1}{4 q_{1}^{3}}\left(p_{3}+C\right)^{2}+\frac{K_{2}}{q_{1}^{3}}-\frac{2 K_{1} q_{1} q_{2}^{2}}{\sin ^{2}\left(2 q_{3}\right)}, \\
& \dot{p}_{2}=\frac{1}{4 q_{2}^{3}}\left(p_{3}-C\right)^{2}+\frac{K_{2}}{q_{2}^{3}}-\frac{2 K_{1} q_{1}^{2} q_{2}}{\sin ^{2}\left(2 q_{3}\right)}, \\
& \dot{p}_{3}=\frac{4 K_{1} q_{1}^{2} q_{2}^{2} \cos \left(2 q_{3}\right)}{\sin ^{3}\left(2 q_{3}\right)} .
\end{aligned}
$$

Note that $q_{4}$ can be obtained by the integration of

$$
\dot{q}_{4}=\frac{\left(q_{1}^{2}+q_{2}^{2}\right) C-\left(q_{1}^{2}-q_{2}^{2}\right) p_{3}}{4 q_{1}^{2} q_{2}^{2}},
$$

after the numerical integration of the previous six differential equations is done. The reduction in the number of equations demonstrates a benefit of using shape variables $\left(d_{1}, d_{2}, \gamma\right)$ and $\phi$ instead of $\left(d_{1}, d_{2}, \theta_{1}, \theta_{2}\right)$.

Since the total energy of the system is conserved, the resulting trajectories depend on the initial conditions of the above differential equations and the speed of the agents are constrained by the initial energy of the system. In addition, $p_{4}=\frac{1}{2}\left(\theta_{1}+\theta_{2}\right)=C$ represents the conserved angular momentum of the two agents. If $C=0$, no rotation occurs in the two-agent system around the target. If $C \neq 0$, the twoagent system rotates around the target with a constant angular velocity.

The equilibrium of the reduced Hamilton equations (26) can be calculated by letting $\dot{\mathbf{q}}=0$ and $\dot{\mathbf{p}}=0$. When $C=$ 0 , the equilibrium of (26) is $p_{1}=p_{2}=p_{3}=0, q_{1}=q_{2}=$ $\left(\frac{K_{2}}{2 K_{1}}\right)^{\frac{1}{6}}$, and $q_{3}=\frac{k}{4} \pi$, in which $k$ is an odd integer. When $C \neq 0$, we calculate that the equilibrium is $p_{1}=p_{2}=0$, 
$q_{3}=\frac{k}{4} \pi$, in which $k$ is an odd integer, $p_{3}=\frac{\left(q_{1}^{2}-q_{2}^{2}\right) C}{q_{1}^{2}+q_{2}^{2}}$, and $q_{1}$ and $q_{2}$ satisfy $C^{2} q_{1}^{4}-2 K_{1} q_{1}^{4} q_{2}^{2}\left(q_{1}^{2}+q_{2}^{2}\right)^{2}+K_{2}\left(q_{1}^{2}+q_{2}^{2}\right)^{2}=0$ and $C^{2} q_{2}^{4}-2 K_{1} q_{1}^{2} q_{2}^{4}\left(q_{1}^{2}+q_{2}^{2}\right)^{2}+K_{2}\left(q_{1}^{2}+q_{2}^{2}\right)^{2}=0$.

Next, let's check the stability of the equilibrium of the system (26). Represent the dynamic equations (26) as $\dot{\mathbf{x}}=$ $f(\mathbf{x})$, in which $x_{1}=q_{1}, x_{2}=q_{2}, x_{3}=q_{3}, x_{4}=p_{1}, x_{5}=p_{2}$, and $x_{6}=p_{3}$. Let $\mathbf{x}_{0}$ represent the equilibrium. Then, linearizing the system (26), we calculate that

$$
\begin{gathered}
A=\frac{\partial f(\mathbf{x})}{\partial \mathbf{x}} \\
\left.\begin{array}{cccc}
0 & 0 \\
-\frac{x_{6}-C}{2 x_{1}^{3}} & 0 \\
-\frac{3\left(x_{6}+C\right)^{2}}{4 x_{1}^{4}}-\frac{3 K_{2}}{x_{1}^{4}}-\frac{2 K_{1} x_{2}^{2}}{\sin ^{2}\left(2 x_{3}\right)} & -\frac{x_{6}-C}{2 x_{2}^{3}} \\
-\frac{4 K_{1} x_{1} x_{2}}{\sin \left(4 x_{3}\right)} & & -\frac{4 K_{1} x_{1} x_{2}}{\sin \left(4 x_{3}\right)} \\
\frac{8 K_{1} x_{1} x_{2}^{2} \cos \left(2 x_{3}\right)}{\sin ^{3}\left(2 x_{3}\right)} & & & \frac{3\left(x_{6}-C\right)^{2}}{4 x_{2}^{4}}-\frac{3 K_{2}}{x_{2}^{4}}-\frac{2 K_{1} x_{1}^{2}}{\sin ^{2}\left(2 x_{3}\right)} \\
0 & 1 & 0 & 0 \\
0 & 0 & 1 & 0 \\
0 & 0 & 0 & \frac{1}{4}\left(\frac{1}{x_{2}^{2}}+\frac{1}{x_{1}^{2}\left(2 x_{3}\right)}\right. \\
\frac{8 K_{1} x_{1} x_{2}^{2} \cos \left(2 x_{3}\right)}{\sin ^{3}\left(2 x_{3}\right)} & 0 & 0 & \frac{\left(x_{6}+C\right)}{2 x_{1}^{3}} \\
\frac{8 K_{1} x_{1}^{2} x_{2} \cos \left(2 x_{3}\right)}{\sin ^{3}\left(2 x_{3}\right)} & 0 & 0 & \frac{\left(x_{6}-C\right)}{2 x_{2}^{3}} \\
-8 K_{1} x_{1}^{2} x_{2}^{2}\left(\frac{1+2 \cos ^{2}\left(2 x_{3}\right)}{\sin ^{4}\left(2 x_{3}\right)}\right) & 0 & 0 & 0
\end{array}\right) .
\end{gathered}
$$

When $C=0$, at $\mathbf{x}=\mathbf{x}_{0}$, i.e., $x_{1}=x_{2}=\left(\frac{K_{2}}{2 K_{1}}\right)^{\frac{1}{6}}, x_{3}=\frac{k}{4} \pi$, in which $k$ is an odd integer, and $x_{4}=x_{5}=x_{6}=0$, we have

$$
\begin{aligned}
& A=\frac{\partial f\left(\mathbf{x}_{0}\right)}{\partial \mathbf{x}} \\
& =\left(\begin{array}{cc}
0 & 0 \\
0 & 0 \\
0 & 0 \\
-3 K_{2}\left(\frac{2 K_{1}}{K_{2}}\right)^{\frac{2}{3}}-2 K_{1}\left(\frac{K_{2}}{2 K_{1}}\right)^{\frac{1}{3}} & -4 K_{1}\left(\frac{K_{2}}{2 K_{1}}\right)^{\frac{1}{3}} \\
-4 K_{1}\left(\frac{K_{2}}{2 K_{1}}\right)^{\frac{1}{3}} & -3 K_{2}\left(\frac{2 K_{1}}{K_{2}}\right)^{\frac{2}{3}}-2 K_{1}\left(\frac{K_{2}}{2 K_{1}}\right)^{\frac{1}{3}} \\
0 & 0
\end{array}\right. \\
& \left.\begin{array}{cccc}
0 & 1 & 0 & 0 \\
0 & 0 & 1 & 0 \\
0 & 0 & 0 & \frac{1}{2}\left(\frac{2 K_{1}}{K_{2}}\right)^{\frac{1}{3}} \\
0 & 0 & 0 & 0 \\
0 & 0 & 0 & 0 \\
-8 K_{1}\left(\frac{K_{2}}{2 K_{1}}\right)^{\frac{2}{3}} & 0 & 0 & 0
\end{array}\right)
\end{aligned}
$$

Solve for the eigenvalues of $A$ by letting $\operatorname{det}(A-\lambda I)=0$. We calculate the eigenvalues of matrix $A$ as $\left(\sqrt{2\left(4 K_{1}^{2} K_{2}\right)^{1 / 3}} i,-\sqrt{2\left(4 K_{1}^{2} K_{2}\right)^{1 / 3}} i,\left(4 K_{1}^{2} K_{2}\right)^{1 / 3} \sqrt{-\left(\frac{2 K_{1}}{K_{2}}\right)^{1 / 3}} i\right.$, $\left.\left.-\left(4 K_{1}^{2} K_{2}\right)^{1 / 3} \sqrt{-\left(\frac{2 K_{1}}{K_{2}}\right)^{1 / 3}} i\right), \sqrt{6\left(4 K_{1}^{2} K_{2}\right)^{1 / 3}} i,-\sqrt{6\left(4 K_{1}^{2} K_{2}\right)^{1 / 3}} i\right)$ The eigenvalues of $A$ are in the imaginary axis. Then, the equilibriums of the system are stable. At the equilibrium, since $\dot{\mathbf{q}}=0$, the kinetic energy $E(\mathbf{q}, \dot{\mathbf{q}})=0$. The total energy of the system is determined by the potential energy $V(\mathbf{q})$, which is calculated as $V(\mathbf{q})=\frac{K_{1}}{q_{1}^{4}}+K_{2} q_{1}^{2}$.

\section{Simulation Results}

We simulate the trajectories of two sensing agents localizing one stationary target by integrating the differential equations (26) given different initial conditions. The target is located at $(0,0)$.

Choose $K_{1}=1, K_{2}=2$. From $q_{1}=q_{2}=\left(\frac{K_{2}}{2 K_{1}}\right)^{\frac{1}{6}}$, we calculate that, when $C=0$, the equilibrium is $q_{1}(0)=$ $q_{2}(0)=5, q_{3}(0)=\frac{k \pi}{4}$, in which $k$ is an odd integer, and $p_{1}(0)=p_{2}(0)=p_{3}(0)=0$. If we let the agents start from the equilibrium, then, the two agents will stay stationary as $t \rightarrow \infty$ since there is no initial kinetic energy. The total energy of the system is determined by $V(\mathbf{q}(0))=K_{1} q_{1}^{4}(0)+\frac{K_{2}}{q_{1}^{2}(0)}=3$.

Next, let's set $q_{1}(0)=q_{2}(0)=1, q_{3}(0)=\frac{\pi}{6}, p_{1}(0)=$ $p_{2}(0)=p_{3}(0)=0$, and the integration time $T=3 s$. Similar to the previous case, the initial kinetic energy $E=0$ and the total energy $H=V+E=\frac{K_{1} q_{1}^{4}(0)}{\sin ^{2}\left(2 q_{3}(0)\right)}+\frac{K_{2}}{q_{1}^{2}(0)}=\frac{10}{3}$. Fig. 2 shows the trajectories of two agents localizing the target. The target is located at $(0,0)$ illustrated by the magenta dot. The two black dots indicate the starting positions of the two agents. Fig. 3 illustrates the potential energy, kinetic energy, the determinant of FIM, and the Hamiltonian of the system using the blue dotted line, red dashed line, green solid line, and magenta dash-dot line, respectively. From the two figures, we observe that, since the agents are not starting from the equilibrium $\left(q_{3}(0) \neq \frac{\pi}{4}\right)$, they do not stay stationary. When they accelerate, the kinetic energy increases, and the potential energy decreases. When the potential energy reaches its minimum value $V_{\min }=3$, the kinetic energy reaches its maximum value $E_{\max }=\frac{1}{3}$, implying the maximum value of the determinant of FIM. In the meantime, the total energy $H$ remains a constant. This observation agrees with the argument that as more energy is spent, more information is expected to be obtained.

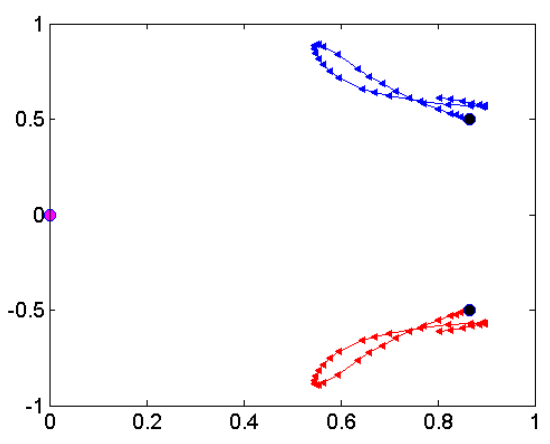

Fig. 2. Trajectories of two agents with the initial condition $q_{1}(0)=q_{2}(0)=$ $1, q_{3}(0)=\frac{\pi}{6}, p_{1}(0)=p_{2}(0)=p_{3}(0)=0, C=0$, and $T=3$.

If we perform the simulation for a longer time interval, for example, $T=10$ instead of $T=3$, the agents just move along the same trajectories back and forth in a similar pattern that they accelerate with increasing information, and decelerate with decreasing information. Their motions are constrained by the initial energy of the system. The simulation results demonstrate a tradeoff between information and energy. 


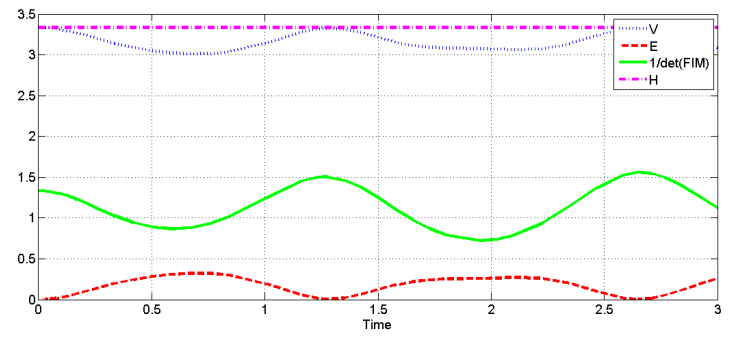

Fig. 3. The kinetic energy, potential energy, determinant of FIM, and Hamiltonian of the tracking system with the initial condition $q_{1}(0)=$ $q_{2}(0)=1, q_{3}(0)=\frac{\pi}{6}, p_{1}(0)=p_{2}(0)=p_{3}(0)=0, C=0$, and $T=3$.

Fig. 4 illustrates the trajectories when $q_{1}(0)=q_{2}(0)=1$, $q_{3}(0)=\frac{\pi}{4}, p_{1}(0)=p_{2}(0)=p_{3}(0)=0$, and $T=3$. In this case, we set $C=1$ instead of $C=0$. We observe that the two-agent system move around the target with fixed angular velocities determined by $C$. Since we let the angular momentum $C$ be positive, the equilibrium is replaced by a limit cycle, to which the motion of the two agents converge. As we can see, the trajectories of the agents depend on a substantial way on initial conditions, which is well-known in classical mechanics.

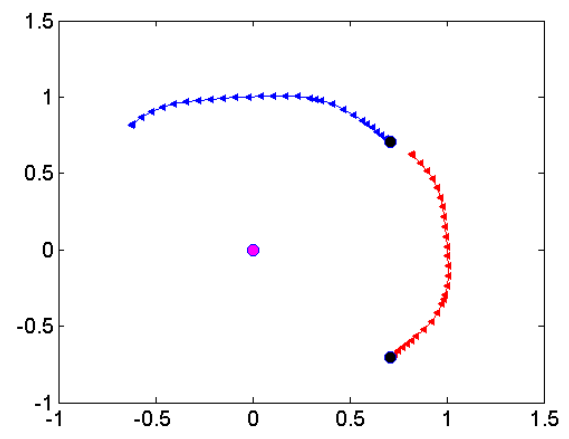

Fig. 4. The trajectories of the two agents with the initial condition $q_{1}(0)=$ $q_{2}(0)=1, q_{3}(0)=\frac{\pi}{4}, p_{1}(0)=p_{2}(0)=p_{3}(0)=0, C=1$, and $T=3$.

\section{CONCLUSIONS AND Future WORK}

Inspired by study in behavioral ecology, this paper initiates an exploration of the tradeoffs between energy and information of mobile agents localizing a target. The determinant of FIM, which is considered as part of potential energy from the insights of classic mechanics, is chosen to represent the quality of information. With the objective of minimizing the difference between the agents' kinetic energy and potential energy, we define an optimal path problem, the resulting trajectories of which satisfy Euler-Lagrange equations, or the Hamilton equations. We evaluate the equilibriums of the resulting dynamic system and demonstrate that the equilibriums are stable. Simulation results with various initial conditions agree with theoretical predictions. Future work includes an extension to tracking a moving target and the incorporation of the state estimation of the target using techniques such as extended Kalman filter and particle filter.

\section{REFERENCES}

[1] J. Ousingsawat and M. E. Campbell, "Optimal cooperative reconnaissance using multiple vehicles," Journal of Guidance, Control, and Dynamics, vol. 30, no. 1, pp. 122-135, 2007.

[2] A. J. Healey, D. P. Horner, S.P.Kragelund, B. Wring, and A. Monarrez, "Collaborative unmanned systems for maritime and port security operations," in Proceedings of the 7th IFAC Conference on Control Applications in Marine Systems, 2007, p. on CD.

[3] S. Martnez and F. Bullo, "Optimal sensor placement and motion coordination for target tracking," Automatica, vol. 42, no. 4, pp. 661$668,2006$.

[4] B. Grocholsky, J. Keller, V. Kumar, and G. Pappas, "Cooperative air and ground surveillance," IEEE Robotics and Automation Magazine, vol. 13 , no. 3, pp. 16-25, 2006.

[5] F. Zhang and N. E. Leonard, "Cooperative control and filtering for cooperative exploration," IEEE Transactions on Automatic Control, vol. 55, no. 3, pp. 650-663, 2010.

[6] Z. Tang and U. Ozguner, "Motion planning for multitarget surveillance with mobile sensor agents," IEEE Transactions on Robotics, vol. 21, no. 5, pp. 898-908, 2005.

[7] M. Wheeler, B. Schrick, W. Whitacre, M. Campbell, R. Rysdyk, and R. Wise, "Cooperative tracking of moving targets by a team of autonomous UAVs," in Proc. IEEE/AIAA 25th Digital Avionics Systems Conf, 2006, pp. 1-9.

[8] B. Bethke, M. Valenti, and J. How, Advances in Cooperative Control and Optimization, 2007, vol. 369, ch. Cooperative Vision Based Estimation and Tracking Using Multiple UAVs, pp. 179-189.

[9] M. E. Campbell and W. W. Whitacre, "Cooperative tracking using vision measurements on seascan UAVs," IEEE Transactions on Control Systems Technology, vol. 15, no. 4, pp. 613-626, 2007.

[10] S. Oh, L. Schenato, P. Chen, and S. Sastry, "Tracking and coordination of multiple agents using sensor networks: System design, algorithms and experiments," Proceedings of the IEEE, vol. 95, no. 1, pp. 234254, 2007.

[11] M. A. MacIver, N. A. Patankar, and A. A. Shirgaonkar, "Energyinformation trade-offs between movement and sensing," PLoS Comput Biol, vol. 6, no. 5, p. e1000769, 2010.

[12] S. A. Stamper, E. Roth, N. J. Cowan, and E. S. Fortune, "Active sensing via movement shapes spatiotemporal patterns of sensory feedback," The Journal of Experimental Biology, vol. 215, pp. 1567$1574,2012$.

[13] P. Skorupski, J. Spaethe, and L. Chittka, "Visual search and decision making in bees: Time, speed, and accuracy," International Journal of Comparative Psychology, vol. 19, pp. 342-357, 2006.

[14] E. J. Warrant, "Seeing in the dark: vision and visual behaviour in nocturnal bees and wasps," The Journal of Experimental Biology, vol. 211, pp. 1737-1746, 2008.

[15] L. Chittka and P. S. ann Nigel E. Raine, "Speedaccuracy tradeoffs in animal decision making," Trends in Ecology and Evolution, vol. 24, no. 7, pp. 400-407, 2009.

[16] S. Hammel, P. Liu, E. Hilliard, and K. Gong, "Optimal observer motion for localization with bearing measurements," Computers \& Mathematics with Applications, vol. 18, no. 1-3, pp. 171-180, 1989.

[17] Y. Oshman and P. Davidson, "Optimization of observer trajectories for bearings-only target localization," IEEE Transactions on Aerospace and Electronic Systems, vol. 35, no. 3, pp. 892-902, 1999.

[18] S. S. Ponda, R. M. Kolacinski, and E. Frazzoli, "Trajectory optimization for target localization using small unmanned aerial vehicles," in In AIAA Guidance, Navigation, and Control Conference, 2009.

[19] K. Doanay and H. Hmam, "Optimal angular sensor separation for AOA localization," Signal Processing, vol. 88, no. 5, pp. 1248-1260, 2008.

[20] A. N. Bishop, B. Fidan, B. D. Anderson, K. Doanay, and P. N. Pathirana, "Optimality analysis of sensor-target localization geometries," Automatica, vol. 46, no. 3, pp. 479-492, 2010.

[21] S. Zhao, B. M. Chen, and T. H. Lee, "Optimal placement of bearingonly sensors for target localization," in Proc. of 2012 American Control Conference, 2012, pp. 5108-5113.

[22] L. N. Hand and J. D. Finch, Analytical Mechanics. Cambridge University Press, 1998.

[23] A. Farina, "Target tracking with bearings-only measurements," Signal Processing, vol. 78, no. 1, pp. 61-78, 1999.

[24] K. Dogancay, "Bearings only target localization using total least squares," Signal Processing, vol. 85, no. 9, pp. 1695-1710, 2003. 